\title{
Los incas y el Sol: métodos de observación solar y calendario incaicos
}

\author{
Elena OrTiz GARCÍA \\ Dpto. de Física \\ Universidad de Alcalá de Henares, Madrid (España) \\ elena.ortizg@uah.es
}

Recibido: 15 de septiembre de 2011

Aceptado: 10 de octubre de 2011

\begin{abstract}
RESUMEN
Algunos de los fenómenos naturales que más han cautivado al ser humano desde la Prehistoria han sido aquellos que ocurren en la bóveda celeste, y por encima de todos, el movimiento periódico del Sol. Los incas no fueron ajenos a ello. En este trabajo se expone el estudio detallado y comparativo de las descripciones en tres cronistas sobre los instrumentos empleados por los incas para realizar observaciones solares. Se analizan las contradicciones que surgen de dicha comparación y su consistencia con las observaciones reales que pueden realizarse desde Cuzco. La resolución de este problema es esencial para comprender el alcance del conocimiento del cielo que poseían los incas y el empleo que hacían de estos conocimientos. Este tipo de observaciones se realizaban con fines de organización social y agrícola a través del establecimiento de un calendario oficial. En el artículo se analizan también las contradicciones en el tipo de calendario utilizado por los incas según diferentes crónicas.
\end{abstract}

Palabras clave: Astronomía incaica, calendario inca, observaciones solares

\section{The Incas and the Sun: Solar Observing Methods and Inca Calendar}

\begin{abstract}
Yet since Prehistory humans have been captivated by the natural phenomena happening across the firmament, and above all the periodic movement of the Sun. Incas also felt this attraction. In this article we carry out a detailed and comparative study of the solar instruments descriptions that appear in three chronicles. We analyze contradictions between them and compared to the real observations that can be carried out in Cuzco. Solving this problem is essential in order to understand which level of astronomical knowledge Incas had achieved and how they applied it. They mainly carried out those solar observations with social and agricultural organization aims and used them in order to fix an official calendar. So, contradictions about Inca calendar between chronicles are also analyzed.
\end{abstract}

Keywords: Inca astronomy, Inca calendar, solar observations.

Sumario: 1. Introducción: El hombre y los fenómenos naturales celestes. 2. Fuentes de información empleadas. 3. Observaciones solares y lunares realizables desde el Cuzco. 4. Análisis de los instrumentos de observación solar incaicos. 5. Empleo de las observaciones solares: el calendario inca. 6. Consideraciones finales. 7. Referencias bibliográficas

\section{Introducción: El hombre y los fenómenos naturales celestes}

Uno de los aspectos de la naturaleza que más han intrigado y perturbado al ser humano desde los albores de la humanidad ha sido el cielo y los objetos que observaba en 
él: los astros. Algunos de ellos parecían permanecer inmutables (las estrellas); otros se movían día tras día, repitiendo sus posiciones al cabo de cierto tiempo: un día, un mes, un año o incluso decenas (el Sol, la Luna y los planetas); y otros aparecían y desaparecían misteriosamente, sin ninguna periodicidad -los cometas y las estrellas fugaces o meteoritos-. Es más, algunos de esos fenómenos celestes parecían tener una relación con la llegada de las épocas lluviosa o seca. Y a otros, por temor a lo desconocido, se les atribuían efectos perniciosos o se les adoraba para conseguir su favor.

Todo ello hizo que fuera el estudio y observación de esa Naturaleza Celeste, la Astronomía, una de las primeras ciencias en desarrollarse en cualquier cultura en la que volquemos nuestro análisis. Todas esas culturas desarrollaron su particular estudio de los astros y su empleo en relación con otros aspectos culturales.

A pesar de que el ser humano, al menos hasta el siglo XX, ha tenido siempre bien anclados los pies en la Tierra, no ha podido substraerse de la belleza y el misterio de la cúpula estelar que le rodeaba y ha tratado de comprenderla y vanamente controlarla.

Sin embargo, al principio y a lo largo de la mayor parte de la historia de la Humanidad, los conocimientos astronómicos se han empleado sólo con un sentido pragmático: en un principio, y sobre todo, con fines predictivos al servicio de la agricultura, y en último caso como subsidiarios al servicio del poder, de aquellos que conseguían al menos predecir el momento de retorno de fenómenos periódicos.

Bien es cierto que ya en Mesopotamia comenzó un estudio en cierto sentido sistemático de los objetos astronómicos, pero continuaba al servicio de una minoría, íntimamente unido a la Astrología (vano y falaz intento del hombre de controlar el futuro). Es realmente en la Grecia clásica cuando comienza una segunda fase, al dar a la observación del cielo un halo científico, y ser los primeros en intentar alcanzar una comprensión del funcionamiento del Cosmos en sí mismo y no por su utilidad calendárica o por ese engañoso intento de predecir un futuro impredecible. Pero no fue hasta los siglos XVI y XVII, y sobre todo, tras las primeras observaciones de Galileo, cuando la Astronomía empezó a considerarse como una ciencia en sí misma, y el estudio de los astros logró tener importancia «per se».

El tema que nos ocupa, la relación de los incas con los fenómenos naturales celestes, con los astros y sus conocimientos astronómicos, y en concreto con el Sol, se enmarca en el primer caso; no parece que llegaran a entrar en esa segunda fase. De hecho, el conocimiento astronómico incaico aparece íntimamente relacionado con las ceremonias religiosas y agrícolas. La observación de los astros era empleada para el mantenimiento correcto del calendario agrícola y festivo, pero además como herramienta de poder de los Incas sobre sus súbditos. El Inca conocía y controlaba, e incluso llegó a vanagloriarse de estar emparentado con el mayor astro del cielo: el Sol. Él dictaba cuándo debía comenzar la siembra y cuándo debía recolectarse.

De cualquier forma, no debemos olvidar que el Imperio Inca que se encontraron los españoles a su llegada y conquista en 1534 era fruto de una larga evolución de pueblos que habitaron previamente en la zona. Por tanto, su cultura, y por ende sus conocimientos astronómicos, estaban imbuidos de todos los desarrollos de culturas anteriores. 


\section{Fuentes de información empleadas}

Para el estudio de los conocimientos astronómicos en la época andina disponemos de varios tipos de fuentes.

La primera y, hasta el momento más fértil, son las crónicas escritas por los españoles en los primeros tiempos tras la conquista, o en contados casos, por indígenas o mestizos. El empleo de estas fuentes de información debe hacerse teniendo siempre muy presente tanto la formación astronómica de estos cronistas como los conocimientos difundidos en la época en España - por ejemplo, los nombres que recibían las constelaciones, o la corrección gregoriana ${ }^{1}$ del calendario, adoptada en Perú el 4 de Octubre de 1584 por orden de Felipe II de 14 de Mayo de 1583, obedecida al año siguiente (Comas 1957)-.

Por otro lado, se debe tener muy presente el acceso a la información sobre la cultura inca que pudieran tener estos cronistas, bien por su conocimiento de las lenguas indígenas, bien por la realización de las investigaciones en fechas tempranas tras la conquista, bien por la interrogación directa a informantes autóctonos. En este sentido cabe resaltar que algunas crónicas no son más que copias tardías de otras anteriores, sin el uso de informantes indígenas directos. Además, siempre hemos de tener en cuenta la finalidad del cronista, dado que ello influirá en los datos y la forma de contarlos que emplee, e incluso a veces, la tergiversación de esos datos.

Aparte de las fuentes escritas, que como hemos dicho son sin duda las que proporcionan el mayor volumen de información, los restos arqueológicos y arquitectónicos pueden proporcinar también información astronómica. La orientación de algunos elementos arquitectónicos nos proporciona indicios de los conocimientos astronómicos e incluso, en ocasiones, algunos de ellos pueden haber sido ubicados y orientados precisamente para un uso relacionado con la Astronomía y en especial con el calendario (v.gr. la obra clave de Bauer y Deaborn 1998).

También los estudios etnográficos en la región nos proporcionan datos importantes, que pueden completar y ayudar en la interpretación de los de las crónicas escritas. En estos estudios hay que tener especial cuidado con los fenómenos de sincretismo con el cristianismo, que se produjeron desde los primeros momentos de la conquista.

Y por último y habiéndose desarrollado sobre todo en los últimos tiempos, el estudio de los quipus (Urton 2007), los «manojos» de cuerdas anudadas que se utlizaban como elementos nemotécnicos en los que se almacenaba información numérica.

1 El calendario que empleamos actualmente en Occidente es el conocido como calendario gregoriano, reforma del calendario juliano introducida a propuesta del papa Gregorio XIII. En el calendario juliano, que venía empleándose desde su introducción por Julio César en el año 46 a.C. (retocado por Augusto), el año contiene 365 días, excepto cada 4 años, que se introducía un día más (año bisiesto). Dado que el año contiene 365,24 días solares medios (el Sol tarda ese tiempo en pasar de nuevo por el equinoccio), en el calendario juliano cada aproximadamente 400 años se produce un desfase de 3 días entre, digamos, el paso del Sol por equinoccio de primavera y el día 21 de Marzo, que nosotros consideramos como día de tal equinoccio. Así, en 1582, las celebraciones religiosas cristianas se habían desplazado 10 días respecto a la posición solar. Esto fue lo que movió a Gregorio XIII a introducir dos tipos de correcciones: (1) al 4 de Octubre le siguió el 15 de Octubre de 1582 y (2) a partir de ese momento los años divisibles por 100 no serían bisiestos, excepto los divisibles por 400 . 
Para la realización de este trabajo se han analizado, sobre todo, fuentes escritas por los cronistas, aunque para algunos aspectos se han consultado datos arqueológicos y etnográficos de otros autores. Se han estudiado especialmente las crónicas de Juan de Betanzos (1987 [1551]), Pedro Sarmiento de Gamboa (2007 [1572]) y el Inca Garcilaso de la Vega (1609). Así mismo, se han empleado dos programas informáticos de simulación astronómica de acceso libre: Stellarium versión 0.10.6, para la simulación de la esfera estelar en Cuzco, y Shadows versión 3.4, para la simulación de relojes solares.

\section{Observaciones solares y lunares realizables desde Cuzco}

Dado que nos centraremos en los instrumentos de observación solar empleados por los incas y su aplicación al calendario, resulta imprescindible, en primer lugar, resumir las observaciones solares y lunares esenciales que se pueden realizar desde latitudes de la zona de Cuzco, capital del Imperio Inca. Las observaciones lunares se han venido empleando en muchas culturas, incluida la nuestra, para establecer el mes en el calendario. Las posiciones y apariencia del Sol y la Luna que se observan son consecuencia del movimiento aparente de estos astros respecto de la Tierra, debido a la combinación de los dos movimientos básicos de ésta alrededor de su propio eje y del Sol.

\subsection{Observaciones solares}

Como se ha indicado, la finalidad básica del conocimiento astronómico entre los incas era el mantenimiento preciso del calendario para la correcta celebración de las ceremonias y la indicación del comienzo de siembra y la recolección de los diversos cultivos esenciales. Por ello, el astro principal era el Sol.

La posición del Sol marca el comienzo de cada estación y se repite regularmente al cabo de un año. De hecho, hoy en día sabemos que ciertamente es la energía recibida del Sol, que varía con una periodicidad anual debido al movimiento de la Tierra alrededor de él, la que marca las variaciones en el clima a lo largo del año.

En las latitudes de la península Ibérica, las posiciones principales del Sol son los dos equinoccios y los dos solsticios. Sin embargo, en la latitud de Cuzco se producen además otros dos fenómenos importantes a tener en cuenta, que son los pasos del Sol por el cénit².

Como es bien sabido, al pasar el Sol por el equinoccio, la duración del día y la noche es la misma. Se producen en torno al 21 de Marzo y el 21 de Septiembre, respectivamente para el comienzo de la primavera y el otoño en el hemisferio Norte, y justo al contrario en el Sur. El Sol sale entonces exactamente por el punto este (acimut $90^{\circ}$ ) y se pone por el oeste (acimut $270^{\circ}$ ) sobre el horizonte. En estas fechas la sombra que produce cualquier cuerpo sobre una superficie horizontal a lo largo de todo el día se mueve en una línea recta que une el este con el oeste.

2 Cénit: punto de la bóveda celeste exactamente en la vertical del observador, encima de su cabeza. 


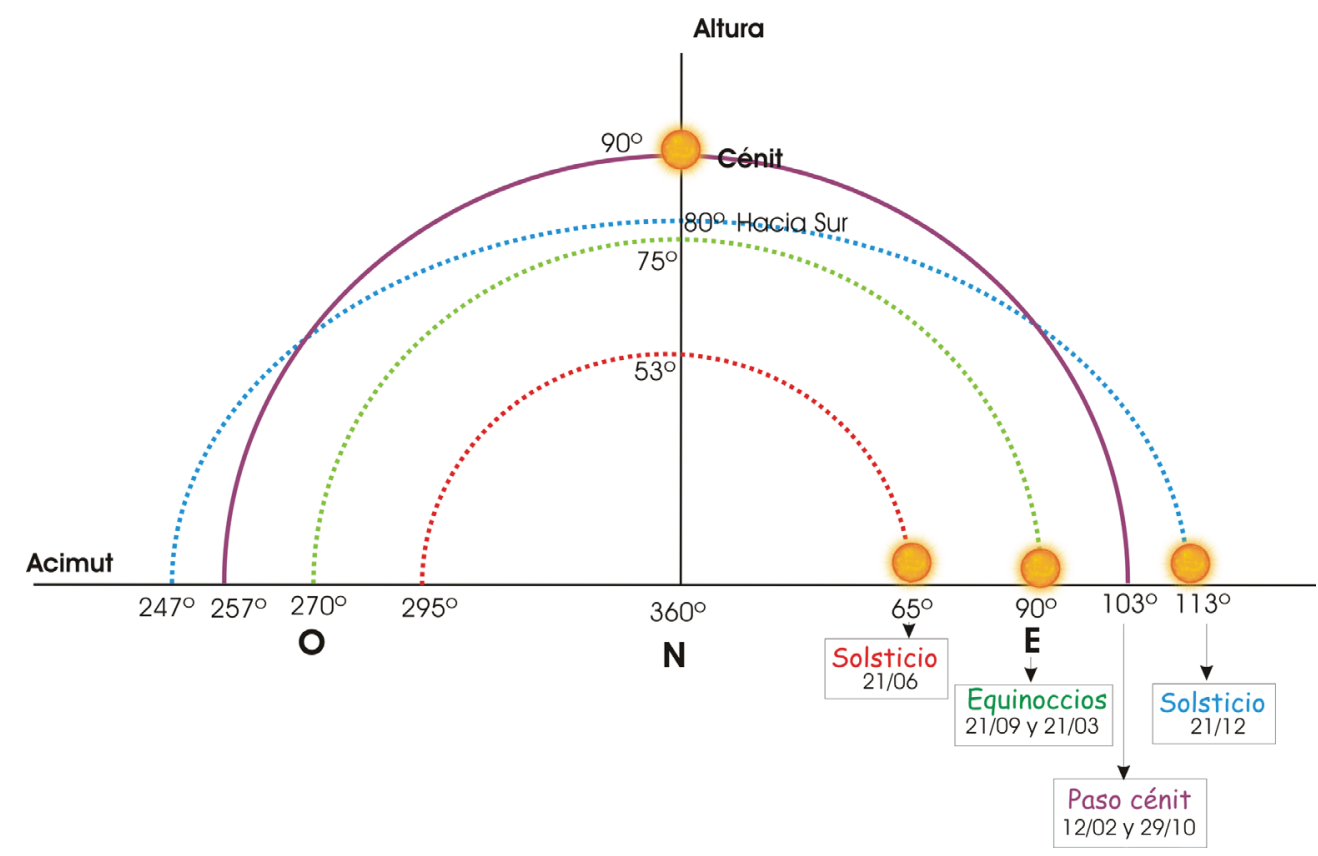

Figura 1: Trayectoria seguida por el Sol para la latitud de Cuzco. Se indica el acimut a lo largo del horizonte y la altura del Sol en grados sobre él para las fechas de los solsticios y los equinoccios y los pasos por el cénit. El 12 de Febrero y el 29 de Octubre el Sol a mediodía no produce sombra. El resto de días sí se produce esa sombra, hacia el Norte desde el 30 de Octubre hasta al 12 de Febrero, incluyendo el 21 de Diciembre, y hacia el Sur desde el 12 de Febrero al 30 de Octubre, incluyendo en equinoccios y solsticio del 21 de Junio. Para esta última fecha, la sombra es la más larga de todo el año a mediodía.

Por el contrario, en los solsticios el Sol alcanza su máxima y su mínima posición sobre el cielo del observador a mediodía solar. Como consecuencia, la noche es la más corta o la más larga del año, respectivamente. Tienen lugar en torno al día 21 de Junio y 21 de Diciembre.

En los pasos del Sol por el cénit, éste no produce sombra al mediodía solar ${ }^{3}$. Estos pasos sólo son observables en zonas de latitudes entre los Trópicos (entre 23 26' 16" $\mathrm{N}$ y S), en las que el astro rey puede alcanzar una altura de $90^{\circ}$ sobre el horizonte en algún día del año. En concreto, para la latitud de Cuzco (1331' 12” S), se producen los días 12-13 de Febrero y 29-30 de Octubre. Este fenómeno coincide con la fecha de los equinoccios sólo en el caso de lugares con latitudes de $0^{\circ}$, esto es, en el círculo máximo conocido como Ecuador, como es el caso de la ciudad de Quito, que también perteneció al Imperio Inca.

3 Eratóstenes, en el siglo III a.C., usó este fenómeno producido en Siena (Asuán, Egipto) para determinar con bastante exactitud el radio terrestre al compararlo con la sombra que se produce en Alejandría y la distancia entre las dos ciudades. 


\subsection{Observaciones lunares}

La Luna presenta también una periodicidad en el cielo, pero, en este caso, más corta. El período que nos interesa es el sinódico de 29,5 días, pues es el más fácilmente observable. La Luna pasa, en ese período, por sus conocidas 4 fases: nueva, cuarto creciente, cuarto menguante y llena. Y vuelve a repetirse cuando el Sol vuelve a iluminar la misma parte de la Luna, esto es, se encuentra en la misma posición respecto del Sol y la Tierra. Hay que remarcar que no coincide con el tiempo que tarda nuestro satélite en completar la vuelta alrededor de la Tierra, que es de aproximadamente 28 días.

El caso es que el mencionado período sinódico es el que se conoce como lunación o mes lunar, y ha servido, en general, para definir el mes en los distintos calendarios ya sean lunares o solares.

\section{Análisis de los instrumentos de observación solar incaicos}

Hasta 1609, año en que Galileo construyó el primer telescopio y lo empleó con fines astronómicos, los estudios de los astros se realizaban a simple vista, o como mucho, con la ayuda de algún sencillo instrumento de medida como el astrolabio, el cuadrante o el gnomon.

Este último, el gnomon, tuvo una aplicación generalizada en muchas culturas antiguas. Se trata del instrumento más simple para construir un reloj y llevar el calendario. Consiste simplemente en un palo o pilar, inclinado o vertical, que permite seguir el movimiento del Sol poniendo atención a la dirección y longitud de la sombra que el astro rey proyecta.

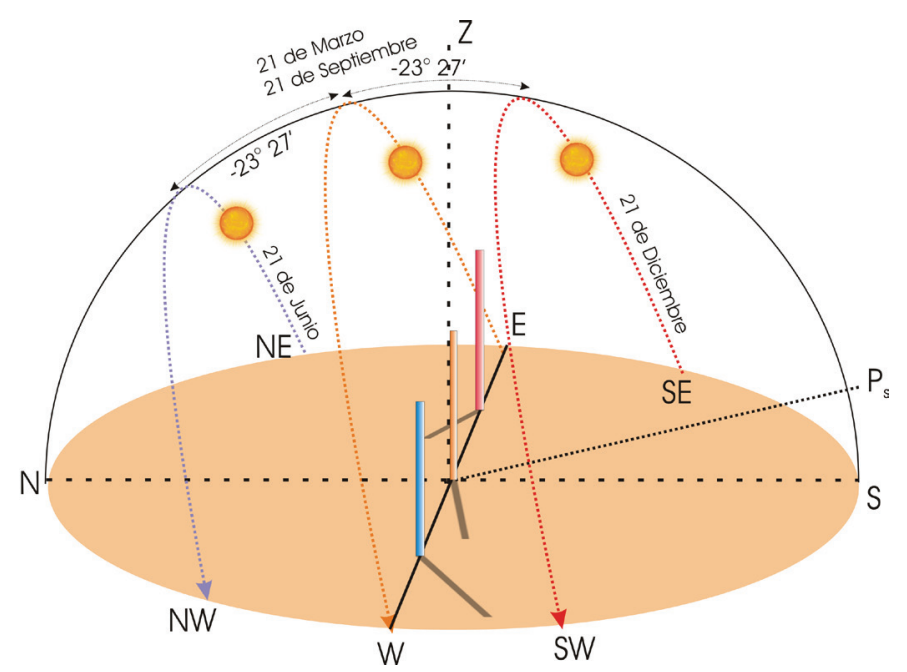

Figura 2: Gnomon para latitud de Cuzco. Cualquier elemento vertical puede ser usado como gnomon. En la figura se observa cómo la sombra que produce un poste vertical varía según la hora del día y el día del año a la que se observe. Por ello puede ser empleado como reloj o cómo instrumento de seguimiento del calendario. 
Más sencillo aún es el empleo de elementos del paisaje o arquitectónicos. Estos elementos, como vanos de puertas o ventanas y columnas, observados desde puntos alejados, marcan la posición del Sol en un momento determinado en un día determinado. Como la posición del Sol varía cíclicamente con la hora y el día del año, ha sido muy empleado ya desde tiempos prehistóricos para hacer coincidir, por ejemplo, ceremonias religiosas con equinoccios o solsticios.

Según algunos cronistas, los incas emplearon este tipo de instrumentos para mantener el calendario, aunque como veremos las informaciones son contradictorias en cierto sentido. Según Polo de Ondegardo (1906 [1559]: cap. VII) y Acosta (1895 [1590]: Libro VI, cap. III) recibían el nombre de sucancas, aunque en el capítulo XI de El mundo de los incas, Polo de Ondegardo los llama saybas. Se trataba de un grupo de pilares colocados en ciertas posiciones en el entorno del Cuzco. Observados desde un cierto lugar, permitían conocer la fecha en el calendario solar por la posición del Sol respecto de estos pilares, a su salida o puesta. Es posible que estuvieran ubicados en dos grupos: uno hacia el Sol naciente y otro hacia el poniente. A pesar de los esfuerzos arqueológicos realizados para su localización, aún no se han podido encontrar, por lo que dependemos únicamente de las fuentes escritas tras la conquista de los españoles para su comprensión.

Varios son los problemas que surgen al estudiar a través de las crónicas estas $\mathrm{su}$ cancas, por otro lado esenciales para conocer el alcance del saber astronómico de los incas.

En primer lugar, no sabemos con seguridad cuál fue su número. Por otro lado, tampoco está muy claro cómo eran estos pilares (su altura y separación), ni su ubicación exacta ni el lugar desde dónde se realizaban las observaciones. Tampoco sabemos con la suficiente certeza qué posiciones clave del Sol eran las que se seguían con estas columnas.

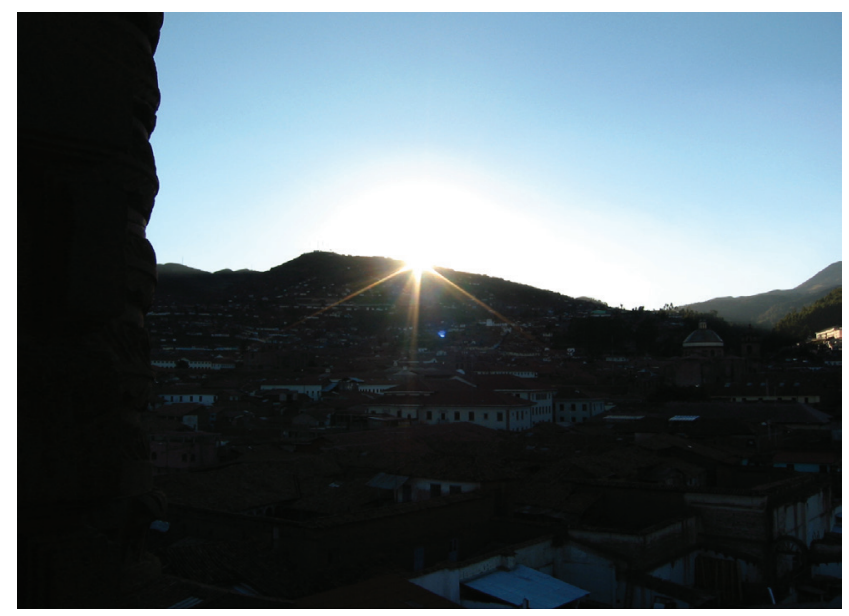

Figura 3: Puesta de Sol desde el campanario de Santo Domingo, Cuzco (24/07/2002). Los elementos del paisaje en el horizonte pueden servir para marcar fechas claves del calendario observando las puestas y salidas del Sol desde un lugar alejado. 
En este trabajo vamos a analizar en profundidad la obra de tres de estos cronistas que, con cierto detalle, proporcionan descripciones incluso contradictorias, dejando para una publicación posterior el análisis pormenorizado y comparativo de todas las informaciones escritas que nos han llegado.

Empecemos por aquello en lo que sí parecen estar de acuerdo los cronistas: fue Inca Pachacutec Yupanqui el que se ocupó de erigir estos pilares en torno a Cuzco. Al menos eso contaban los informantes indígenas al ser preguntados y así nos lo han transmitido las crónicas. Por tanto, debieron ser ubicados entre aproximadamente 1440 y 1470, y aún seguirían en uso al llegar los españoles. Sin embargo, a partir de aquí comienzan las contradicciones.

Cuadro 1: Comparación de la información sobre las columnas de observación solar en los tres cronistas analizados

\begin{tabular}{lcccclll}
\hline Cronista & $\begin{array}{c}\mathbf{N}^{\mathbf{0}} \text { grupos } \\
\text { Este Oeste }\end{array}$ & $\begin{array}{c}\text { Torres } \\
\text { por } \\
\text { grupo }\end{array}$ & $\begin{array}{c}\text { Separación } \\
\text { entre los inte- } \\
\text { riores }\end{array}$ & $\begin{array}{c}\text { Altura } \\
\text { exteriores }\end{array}$ & $\begin{array}{c}\text { Altura } \\
\text { interiores }\end{array}$ & Observación \\
\hline Betanzos & 1 & 1 & 4 & $\begin{array}{l}0,5 \text { brazas }= \\
0,83 \mathrm{~m}\end{array}$ & $\begin{array}{l}\text { Mayores que } 2 \text { estados }= \\
\text { interiores } \\
\text { Mayores que } \\
\text { interiores, } \\
\text { comparables }\end{array}$ & $\begin{array}{l}\text { Todos los días } \\
\text { a atalayas }\end{array}$ \\
Garcilaso & 2 & 2 & 4 & $\begin{array}{l}18-20 \text { pies }= \\
5,56 \mathrm{~m}\end{array}$ & Solsticios \\
Sarmiento & 1 & 1 & & $\begin{array}{l}2 \text { varas }=1,67 \\
\mathrm{~m}\end{array}$ & & \\
\hline
\end{tabular}

Analizaremos en primer lugar la Suma e narración de los incas de Juan de Betanzos, que en época tan temprana tras la conquista como es 1551, proporciona una de las primeras visiones detalladas de tales elementos. La importancia de este cronista reside no sólo en lo temprano de sus escritos, sino en que además vivió mucho tiempo en Cuzco y estuvo casado con la viuda de Atahualpa, por lo que debió ver facilitado su acceso directo a la información de los indígenas. Él nos cuenta que:

«Y tras esto se ponia, cuando se ponia el sol, en cierto sitio, en el cual estuvo seguro en pié en una parte donde bien ver se pudiese, y ansí como conociese desde aquel sitio do él se paraba, el curso por do el sol iba cuando se ponia, en aquel derecho, en lo más alto de los cerros, hizo hacer cuatro pirámides ó mármoles de cantería, los dos en medio menores que los otros dos de los lados, y de dos estados ${ }^{4}$ de altor cada uno, cuadrados, é apartado uno de otro una braza ${ }^{5}$, salvo que los dos pequeños de en medio hizo más juntos, que del uno al otro habrá media braza. Y cuando el sol salía, estando uno puesto do inca Yupanqui se paró para mirar y tantear este derecho, sale y va por el derecho y medio destos dos pilares, y cuando se pone, lo mismo, por la parte do se pone; por donde la gente común tenia entendimiento del tiempo que era, ansí de sembrar, como

\footnotetext{
${ }^{4}$ Un estado, según la Real Academia de la Lengua, corresponde a la estatura de un hombre, es decir, 7 pies o 3,9 m, dado que un pie correspondía a unos $27,8 \mathrm{~m}$.

5 Braza: la distancia media entre los dedos pulgares del hombre, extendidos horizontalmente los brazos; equivalente a 2 varas, es decir , 1,67 m.
} 
de coger; porque los relojes eran cuatro á do el sol salía, y otros cuatro á do se ponia, do se diferenciaban los transcursos y movimientos que así el sol hace en el año» (Betanzos 1987 [1551]: cap. XV).

Y un poco más adelante nos dice:

«y porque andando el tiempo no perdiesen la cuenta de estos meses y los tiempos que habia de sembrar y hacer las fiestas, que ya les habia dicho que habia hecho aquellos pachaunanchac ${ }^{6}$, que dice relojes, los cuales habia hecho en estos diez dias que se tardó en no les querer declarar lo que ya habéis oido; los cuales relojes es desta manera: Que todas las mañanas é tardes miraba el sol en todos los meses del año mirando los tiempos del sembrar y cojer, y ansímismo cuando el sol se ponia; y ansímismo miraba la luna cuando era nueva é llena é menguante; los cuales relojes hacia hacer encima de los cerros más altos á la parte do el sol salia y á la parte donde se pone» (Betanzos 1987 [1551]: cap. XV).

Como vemos, Betanzos indica que eran 4 los pilares colocados a la puesta de Sol y otros 4 a la salida, e incluso da cuenta de sus medidas y de la distancia entre ellos. El problema es que no precisa ni el lugar donde estaban colocados, ni el lugar desde donde se observaban. Lo único que constata es que estaban en lo alto de los cerros más altos. Según Betanzos, los «relojes», a los que llama pachaunanchac, debían ser observados todos los días de todos los meses del año, a la salida y puesta del Sol.

También Cieza de León se refiere, aunque muy escuetamente, al empleo de los pilares, pero afirma que había muchos ubicados en los cerros en torno a Cuzco:

«Por otra estaba el cerro de Carmenga, de donde salen a trecho ciertas torrecillas pequeñas, que servían para tener cuenta con el movimiento del sol, de que ellos mucho se preciaron». (Cieza de León 2005 [1554]: cap. XCII).

«Y usaron de unas torrecillas pequeñas, que hoy día están muchas por los collados del Cuzco, algo ahusadas, para por la sombra que el sol hacía en ellas entender en las sementeras y en lo que ellos más sobre esto entienden». (Cieza de León 2005 [1554]: cap. XXVI).

Ahondando en el mismo tipo de pilar encontramos la descripción de Garcilaso de la Vega en sus Comentarios Reales (1609: Primera parte, libro segundo, cap. XXII), que además nos proporciona más detalles (no la reproducimos aquí textualmente dada su longitud). Sin embargo, este cronista nos habla de ocho torres al oriente y ocho al poniente de la ciudad de Cuzco, colocadas de cuatro en cuatro. Dos de ellas, las pequeñas, medían 3 estados de alto y estaban separadas 18 o 20 pies entre sí. Se colocaban en medio de dos más grandes que distaban también 18 o 20 pies de las pequeñas.

Como vemos, Garcilaso las describe más altas y más separadas entre sí que Betanzos, aunque ambos coinciden en que estaban colocadas equidistantes entre sí.

Garcilaso de la Vega deja claro que las dos exteriores sólo servían para poder localizar desde lejos las dos más pequeñas con facilidad. Lo mismo parece extraerse de Betanzos, puesto que lo que describe es el paso del Sol por las centrales.

6 Pachaunanchac: «señaladores de tiempo» (pacha: tiempo; unancha: señal; diccionario AULEX de quechua: http://aulex.org/qu-es/). 


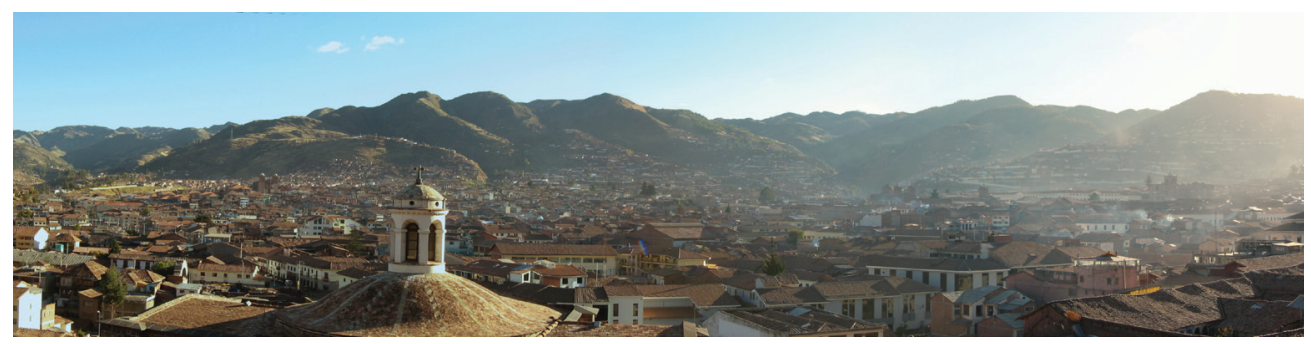

Figura 4: Cerros en el entorno de Cuzco desde el campanario de Santo Domingo. Esta vista está dirigida hacia el O-SO. El Oeste está muy próximo al extremo derecho de la foto, y se aprecia la puesta de Sol un poco más al Norte del Oeste.

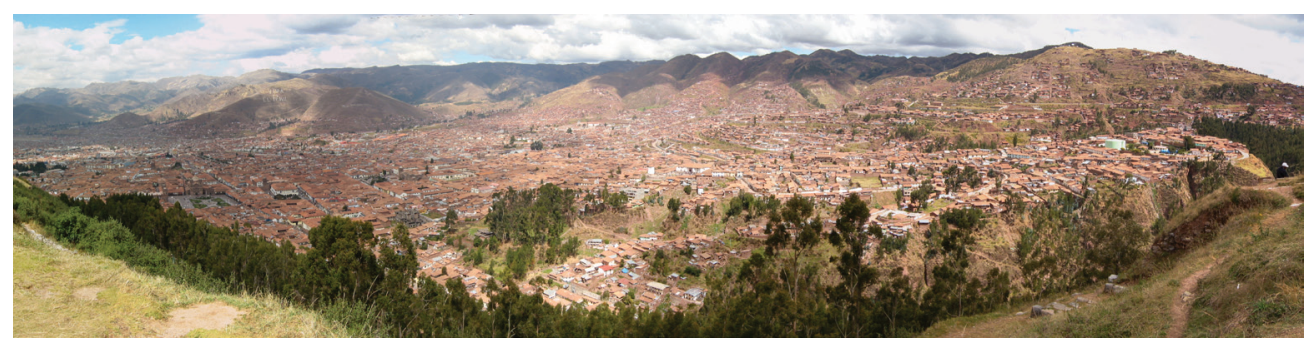

Figura 5: Vista panorámica de Cuzco y su entorno desde Sacsayhuaman en dirección Sur (salida del Sol a la izquierda de la fotografía y puesta a la derecha).

Así, es de destacar que el sistema de verificación creado, al redundar las columnas al este y al oeste para la misma fecha y dado que siempre que se realizan este tipo de observaciones, puede incurrir en un error de algún día, pues la posición del Sol no varía mucho de un día a otro. Esta comprobación servía para asegurar la correcta determinación del día, pero no sólo eso, el sistema permite que si al amanecer la zona del este estuviera cubierta de nubes o niebla, al anochecer se tuviera una nueva oportunidad de observación.

Existe una diferencia clara e importante entre ambas descripciones, la de Betanzos y la de Garcilaso. Éste último constata que se usaban sólo para determinar la fecha de los solsticios, observando el paso del Sol por las columnas centrales a la salida en las que estaban colocadas al este y en la puesta en las que estaban colocadas al oeste. Por el contrario, Betanzos asegura que se observaban todos los días.

Parece más realista la observación sólo de los solsticios, porque para poder determinar la salida del Sol todos los días del año debería de haber más columnas a lo largo del horizonte, ocupando un total de aproximadamente $48^{\circ}$ de acimut sobre el horizonte, que es la variación que presenta en su salida y puesta el Sol a lo largo de todo el año.

Lo interesante de Garcilaso es que no habla de oídas, sino que menciona que él mismo había visto estos pilares y que los dejó en pie en 1560. No pudo llegar a saber desde dónde se realizaban las observaciones, aunque deja entrever que podría ser desde el Co- 
ricancha (actual templo de Santo Domingo, en Cuzco), pero señaló que podrían hacerse las comprobaciones necesarias sabiendo que lo que se observaban eran los solsticios.

Garcilaso añade que Cieza (2005 [1554]: cap. XCII), como hemos visto más arriba, y Acosta (1894 [1590]: Libro VI, cap. III) hacen mención de estas columnas en sus crónicas, pero que no acertaron a entender su empleo: de ahí la vaguedad en la descripción realizada por dichos autores.

Por último, mencionaremos a Pedro Sarmiento de Gamboa, que realiza una descripción muy distinta sobre el empleo de las torres, aunque con mucho detalle:

«Y para que el tiempo del sembrar y del coger se supiese precisamente y nunca se perdiese, hizo poner en un monte alto al levante del Cuzco cuatro palos, apartados el uno del otro como dos varas de medir, y en las cabezas de ellos unos agujeros por donde entrase el sol a manera de reloj o astrolabio. Y considerando a donde hería el sol por aquellos agujeros al tiempo del barbechar y sembrar, hizo sus señales en el suelo, y puso otros palos en la parte que corresponde al poniente del Cuzco para el tiempo de coger las mieses. Y como tuvo certificados estos palos precisamente, puso para perpetuidad en su lugar unas columnas de piedra de la medida y agujeros de los palos, y a la redonda mando enlosar el suelo, y en las losas hizo hacer ciertas rayas niveladas conforme a las mudanzas del sol, que entraba por los agujeros de las columnas de manera que todo era un artificio de reloj anual, por donde se gobernaban para el sembrar y coger. Y diputó personas que tuviesen cuenta con estos relojes y notificasen a1 pueblo los tiempos y sus diferencias, que aquellos relojes señalasen.» (Sarmiento de Gamboa 2007 [1572]: cap. XXX).

En este breve pasaje aparecen diferencias esenciales en la forma de usar estas columnas. No debía observarse el Sol desde otro lugar en el Cuzco, sino que debían «leerse» sus datos «in situ». El propio suelo, a los pies de las columnas, estaba enlosado y marcado.

Además, la forma de usar los relojes es totalmente distinta de la que se infiere de los textos de Betanzos y Garcilaso. En la descripción de Sarmiento de Gamboa, los pilares presentaban unos agujeros en la parte superior que eran atravesados por los rayos del Sol, y el rayo incidía en las marcas hechas ex profeso en el suelo a su alrededor. Cada marca indicaba el tiempo adecuado de una siembra o del barbecho. Y otro dato más: nos dice que los del levante se usaban para la siembra y el barbecho y los del poniente para la cosecha.

Nos indica además este autor que había personas especializadas, dedicadas exclusivamente a la atención de estos «relojes» y a notificar en su momento al pueblo la tarea que debían realizar.

Por otro lado, podemos inferir que el método era bien conocido, porque usaban primero una especie de modelo, los palos, y posteriormente se construía el «reloj anual» definitivo, en columnas de piedra de la misma medida y con agujeros similares.

Pedro Sarmiento de Gamboa era cosmógrafo y matemático, lo que nos sugiere que podría haber captado con cierta facilidad el sistema empleado por los incas. Sin embargo, también es posible que ese mismo conocimiento le llevara a añadir información de su propia cosecha al oír hablar de tales columnas. De hecho, no tendría mucho sentido emplear cuatro columnas en el mismo sitio para usarlas como gnomon: con una sería más que suficiente. Es mucho más lógica la justificación que proporciona 
Garcilaso para el empleo de grupos de cuatro columnas: las dos mayores exteriores servirían de localizadores para las otras dos menores, las cuales se emplearían para certificar el día que el Sol pasa entre ellas.

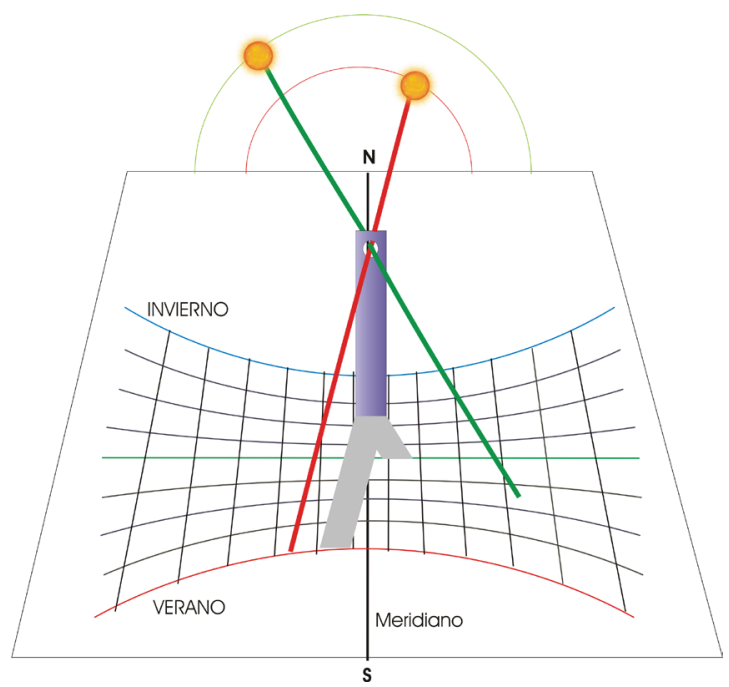

Figura 6: Esquema representativo del sistema de observación descrito por Sarmiento de Gamboa. Según su descripción los pilares no se usaban siguiendo la posición de su sombra, sino que los rayos del Sol atravesaban unos agujeros que se habían realizado en la columna y la atravesaban, incidiendo sobre unas marcas en el suelo. En el gráfico se han representado las líneas sobre las que se movería la sombra a lo largo del día en diferentes fechas del año. La central en verde corresponde a los equinoccios.

De hecho, el propio Garcilaso de la Vega (1609: cap. XXII) da cuenta de la existencia de otros pilares que sí eran usados como el gnomon descrito al comienzo de este apartado. Éstos estaban ubicados en plazas y patios en los templos del Sol (en Cuzco, sería ante el Coricancha), rodeados de un cerco redondo, y servían, según nos cuenta, principalmente para indicar los equinoccios. Por su descripción debían considerarse muy importantes, puesto que estaban ricamente labrados.

El sistema era sencillo: ante la proximidad del equinoccio los sacerdotes encargados seguían la sombra de la columna a lo largo de todo el día, desde el amanecer al anochecer. Extendían un hilo de este a oeste, puntos cardinales de los que por experiencia ya conocían exactamente su ubicación. Cuando la sombra seguía este hilo, sin salirse de él, durante todo el día, sabían que había llegado el equinoccio correspondiente.

Garcilaso señala así mismo que estas columnas se ubicaban en todos los sitios del Imperio Inca, en las plazas, y que en Quito se situaba la más venerada, pues el equinoccio en este lugar coincide con el paso del Sol por el cénit, y por tanto la columna no produciría sombra y la luz del Sol la bañaría por completo. Por desgracia, también cuenta que los españoles mandaron destruirlas todas ellas por ser huacas muy veneradas por los indígenas. 
En conclusión, del análisis de estos tres cronistas parece extraerse que existieron diversos tipos de columnas para realizar observaciones solares. Lo más probable es que las ubicadas en los cerros del Cuzco se emplearan sólo para algunas posiciones solares clave, seguramente los solsticios para fijar el calendario solar. El otro tipo que menciona Garcilaso se emplearía sobre todo en el equinoccio.

Para poder cerciorarnos de la consistencia de cada una de las versiones analizadas, sería esencial poder localizar los pilares con estudios arqueológicos. Hasta la fecha, todos los intentos realizados en el Cuzco han resultado infructuosos (Bauer y Deaborn 1998).

Entretanto, resulta imprescindible ampliar este trabajo realizando un estudio minucioso de todas las fuentes escritas disponibles.

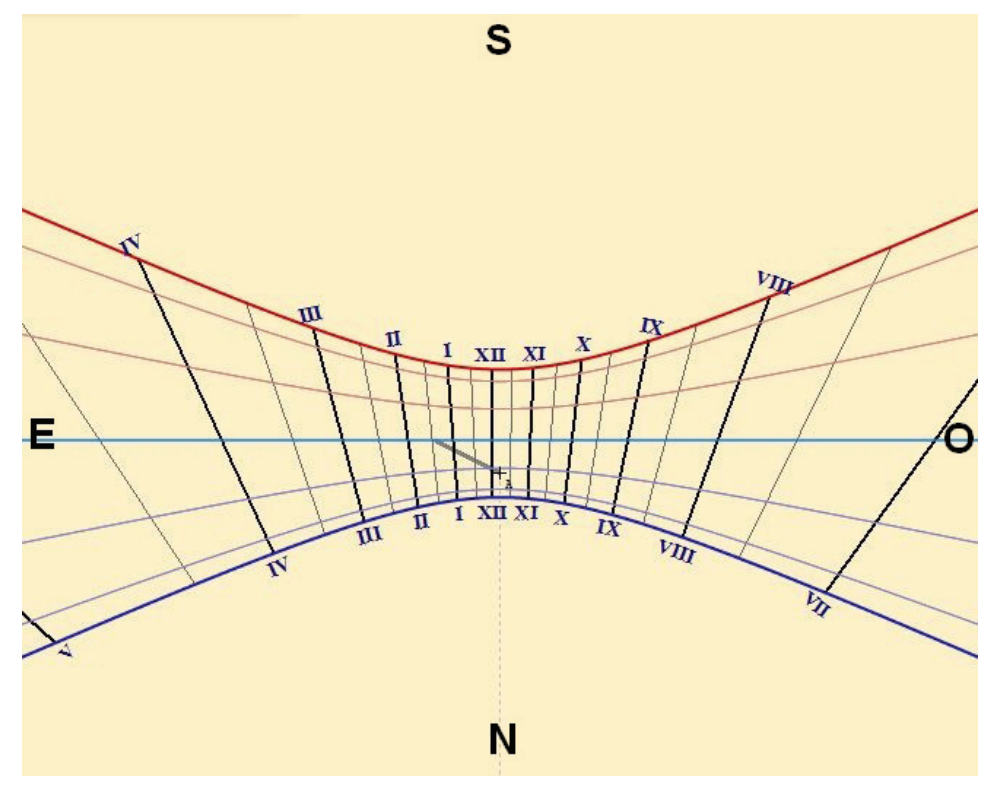

Figura 7: Reloj solar horizontal para coordenadas geográficas de Cuzco. Si estas líneas estuvieran dibujadas en el suelo de un lugar de Cuzco, con el Sur en la parte superior, y se colocara un pilar vertical en el lugar marcado como A, en los días de equinoccio, el extremo de la sombra del pilar seguiría la línea horizontal en azul claro, desde la salida la puesta de Sol. En el solsticio de Diciembre, el extremo de la sombra recorrería la línea azul oscuro y en el de verano la roja. La línea que pasa por A sería la recorrida en los días de paso por el cénit, de forma que a mediodía no se produciría sombra. (Obtenida por la autora empleando el programa Shadowspro versión 3.4).

\section{Empleo de las observaciones solares: el calendario inca}

Como hemos indicado, las observaciones realizadas con las columnas analizadas en el apartado anterior servían para mantener el calendario. De hecho, Betanzos afirma que eran observadas todos los meses para que el calendario de ceremonias estuviera 
bien acompasado con el movimiento solar anual. Pero, ¿qué tipo de calendario usaron los incas? ¿Solar, lunar, luni-solar?

Aún hoy esta cuestión continúa en discusión. Todo parece indicar que con la expansión del Imperio se oficializó un calendario solar, pero es posible que simultáneamente y como reminiscencia de épocas anteriores, se empleara un calendario lunar y que probablemente continuara empleándose tras la conquista, dado que estaba mucho más arraigado en la población andina que el impuesto por los incas unos cien años antes de la llegada de los españoles (Bauer y Dearborn 1998; Zuidema 2010). De hecho, los cronistas constatan la existencia de otros calendarios en otras zonas distintas de la capital del Imperio Inca, por ejemplo, los pueblos de la zona del lago Titicaca (Cieza de León 2005 [1554]: Primera parte de la Crónica del Perú, cap. CI).

Betanzos, en su crónica de 1551, nos indica que fue Inca Pachacutec Yupanqui (1438-1471) quien, en su reorganización del Estado incaico, instauró el calendario oficial solar y sus correspondientes celebraciones para todo el Imperio: «inca Yupanqui les dijo que él había muchos años que había imaginado los meses é tiempo del año, los cuales había hallado que eran doce» (Betanzos 1987 [1551]: cap. XV).

También otros autores hacen referencia a este inca como el instaurador del calendario de 12 meses (p. ej. Sarmiento de Gamboa [2007 (1572)]).

En la descripción que realiza Betanzos en el capítulo XV de su obra, se constatan dos aspectos claves del empleo de la Astronomía entre los incas como ciencia subsidiaria al servicio del poder. Por un lado, la estrecha relación entre el calendario y las ceremonias religiosas, puesto que el establecimiento de los meses por el Inca Pachacutec Yupanqui y la enumeración que nos hace el cronista de los distintos meses, no se realiza por la importancia en sí de dicho calendario, sino con el fin de establecer la cronología de las celebraciones a realizar en cada período del año. Por otro lado, estas celebraciones, en las que de una forma u otra participaban todos los habitantes del Imperio, permitían al Inca mostrar su poderío y hacer alarde de su control sobre la organización del Estado, e incluso en algunas de ellas, sobre los fenómenos naturales.

En cuanto al número de días de cada mes, Betanzos afirma que eran 30, lo que daría un total de 360 días al año: «diciendo á estos señores que cadal mes destos tenia treinta días, y que el año tenia trescientos y sesenta» (Betanzos 1987 [1551]: cap. $\mathrm{XV})$.

Dado que el año solar es de aproximadamente 365 días, esto produciría un desfase de 5,25 días cada año entre las celebraciones y la posición del Sol. Sin embargo, aunque no lo menciona explícitamente, parece extraerse de su descripción posterior que los incas iban corrigiendo este desfase guiándose por las columnas descritas en el apartado anterior para indicar el comienzo del año.

Pero el empleo de meses de 30 días no está tan claro, pues otros autores indican que los meses eran lunares. Por ejemplo, Cieza de León menciona que el año (huata) entre los incas constaba de 12 lunas (quilla $)$ : «Contaron el año por ello, al cual llaman «guata» y los hacen de doce lunas, teniendo su cuenta en ello» (Cieza de León 2005 [1554]: cap. XXVI).

\footnotetext{
7 Quilla se emplea en quechua tanto para la Luna como para el mes.
} 
Esto haría un año de 354 días, dado que, como ya hemos dicho, la Luna pasa por la misma fase cada 29,5 días. De todas formas, acto seguido, también Cieza de León nos dice que esta cuenta la llevan por las observaciones del Sol.

Por tanto, no queda muy claro si realmente se seguía a la Luna o el movimiento del Sol para el cómputo de los meses y los años. Es probable que mantuvieran meses lunares y realizaran las correcciones sobre la marcha para hacer coincidir el comienzo del año con la posición adecuada del Sol, que se repite cada aproximadamente 365 días. De hecho, esto es exactamente lo que explica Garcilaso de la Vega (1609: Libro II, cap. XXII). Según él los años constaban de 12 lunas, y para ajustarlo con el movimiento solar tenían las columnas ubicadas alrededor del Cuzco, en coincidencia con Betanzos.

El problema por tanto reside, como bien remarcaron Ziolkowsky y Sadowsky (1982), en el estudio del tipo de mes empleado, si era lunar y si todos los meses tenían los mismos días, y en saber cuándo se incorporaban los días en exceso, y no tanto en el tipo de año empleado, sobre lo cual parece existir un consenso entre los cronistas en que era solar.

Polo de Ondegardo en sus Errores y Supersticiones de los Indios (1906 [1559]), en el capítulo VII, dice explícitamente que esos días en exceso se añadían al último mes, y que era la observación del solsticio del 21 de diciembre el que marcaba el comienzo de un nuevo año. En ese caso, no todos los meses podían tener los mismos días.

En realidad este empleo de varios calendarios simultáneamente no debería causarnos tanta sorpresa, puesto que ha sido verificado en otras culturas americanas antiguas como es el caso de los mayas y los mexicas en México, e incluso en nuestra cultura mantenemos esta simultaneidad. Recordemos cómo la celebración de la Pascua católica, y como consecuencia la del Carnaval, van cambiando de fecha sobre el calendario civil solar que usamos, dado que dicha festividad se rige por el calendario lunar.

En conclusión, parece evidente que los pilares alrededor del Cuzco eran empleados para establecer el comienzo del año y fijar el calendario solar estatal. Sin embargo, la naturaleza de los meses y la probable existencia de otros calendarios religiosos requiere de un estudio más pormenorizado de las fuentes, e incluso de la ayuda de estudios etnográficos.

\section{Consideraciones finales}

Del estudio detallado de las crónicas se observa que existen serias contradicciones en las versiones que se ofrecen sobre cómo y con qué finalidad se realizaban las observaciones solares en el Imperio Inca. La comparación con las observaciones reales que se pueden realizar desde Cuzco nos lleva a pensar que la versión de Garcilaso de la Vega parece la más consistente. Sin embargo, sería de sumo interés extender este trabajo a todas las crónicas disponibles, pues estas observaciones fueron esenciales en la organización social y agrícola del Imperio a través del establecimiento de un calendario oficial. Sobre este último también surgen serios interrogantes y contradicciones al analizar las distintas crónicas que llevan a concluir, en línea con otros investigadores, la existencia simultánea de distintos tipos calendarios. 
De cualquier forma, resulta evidente que el Imperio Inca había alcanzado un cierto conocimiento y comprensión de uno de los fenómenos naturales que más pronto cautivaron al ser humano: el periódico movimiento solar. Y este conocimiento le resultó muy útil para la organización social, a través del establecimiento de las fechas apropiadas para las tareas agrícolas de siembra y cosecha y de las celebraciones que las acompañaban, en las que la élite mostraba su poder y control.

\section{Referencias bibliográficas}

Acosta, José de

1894 Historia natural y moral de las Indias [1590]. Madrid: Imprenta de Ramón Angés.

BAUER, Brian y David S. P. DeARnBorn

1998 Astronomía e Imperio en los Andes. Cuzco: Centro de Estudios Regionales Andinos «Bartolomé de las Casas».

Betanzos, Juan de

1987 Suma y narración de los incas [1551], edición de $\mathrm{M}^{\mathrm{a}}$ del Carmen Martín Rubio. Madrid: Ediciones Atlas.

Cieza de León, Pedro de

2005 Crónica del Perú. Señorío de los incas [1554], edición de Franklin Pease G.Y. Caracas: Biblioteca Ayacucho.

Comas, Juan

1957 «El calendario Gregoriano en América». Historia Mexicana 7 (2): 207-215.

Garcilaso de la Vega, El Inca

1609 Primera parte de los Commentarios reales que tratan del origen de los Yncas, Reyes que fueron del Peru ..., y de todo lo que fue aquel Imperio y su Republica, antes que los Españoles passaran a el [Texto impreso] / escritos por el Ynca Garcilasso de la Vega, natural del Cozco .... Lisboa: Officina de Pedro Crasbeeck. Documento electrónico, <http://bibliotecadigitalhispanica.bne.es:80/webclient/ DeliveryManager?pid=1864842\&custom_att_2=simple_viewer>, con acceso el 20/12/2011.

Polo DE ONDEGARDo, Juan

1906 «Errores y supersticiones de los indios» [1559], edición de Carlos Romero. Revista Histórica 1: 207-231. Lima.

Sarmiento de Gamboa, Pedro

2007 Historia de los incas [1572], edición de Ramón Alba. Madrid: Miraguano Ediciones - Ediciones Polifemo.

URTON, Gary

2007 «A Multi-Year Tukapu Calendar», en Skywatching in the Ancient World: New Perspectives in Cultural Astronomy, Clive Ruggles y Gary Urton, eds., pp. 245268. Boulder: University Press of Colorado.

Zı́́LKowSKI, Mariusz y Robert M. SADOwSKI

1982-84 «Los problemas de la reconstrucción de los calendarios prehispánicos andinos». Estudios Latinoamericanos 9: 45-87. Varsovia. 
ZuideMA, Tom

2010 El calendario inca. Tiempo y espacio en la organización ritual del Cuzco. La idea del pasado. Lima: Fondo Editorial del Congreso del Perú - Pontificia Universidad Católica del Perú. 